\title{
THE EFFECT OF FROZEN IRRADIATED BONE HOMOGRAFTS ON THE HEALING OF DEFECTS IN THE MANDIBLE AND FEMUR OF THE RAT
}

\author{
P. E. Cleaton-Jones, D. H. Retief and G. Maier*, Johannesburg, South Africa
}

From the Dental Research Unit of the University of the Witwatersrand and South African Medical Research Counci and the *Tissue Bank and Transplantation Research Unit of the South African Institute for Medical Research, Johannesburg, South Africa

Numerous studies have been carried out to determine the effect of various types of bone transplants on the healing pattern of experimentally produced defects in animals (Maatz, Lentz and Graf 1954; Burwell 1963; Heipel, Chase and Herndon 1963; Melcher and Irving 1963; Buring and Urist 1967; Raina 1968). These have repeatedly shown that autogenous bone grafts are better than homogenous and heterogenous bone grafts.

In clinical practice it is not always possible to use autogenous grafts, and in such instances homogenous grafts are the next best variety. The advent of tissue banks has made readily available stored homogenous bone which is often irradiated for sterilisation. Since March 1964 some 1,000 cases have been treated using frozen homogenous bone prepared by the Johannesburg Tissue Bank.

This study is an experimental investigation of the effect of frozen irradiated homogenous bone prepared by the Johannesburg Tissue Bank on the healing mechanism in circumscribed defects in albino rats.

\section{MATERIALS AND METHODS}

Preparation of the experimental homografts-Wistar strain albino rats, selected at random, were killed by coal gas. Their femora were immediately excised, cleaned of all soft tissue and cartilage and cut into conveniently sized portions. These were repeatedly washed in normal saline at 37 degrees Centigrade until completely clean. After this, the fragments were individually packed and sealed in polyethylene bags, placed in dry ice at minus 79 degrees Centigrade and gamma irradiated ${ }^{*}$ at this temperature using a ${ }^{60} \mathrm{Co}$ source to a total dose of between four and seven megarads. The irradiated frozen bone was then stored in a deep freeze at minus 30 degrees Centigrade until required. The grafts were thawed at room temperature immediately before use.

Operative procedures-The recipients were forty Wistar strain albino rats of a mean weight of 150 grammes. Under neurolept anaesthesia (a fluanisone-phentanyl mixture, PhilipsDuphar, $0 \cdot 2$ millilitre per 100 grammes body weight) circumscribed defects were made in the mandibles and femora (Fig. 1) as follows.

Mandibles-A longitudinal midline incision two centimetres long was made in the skin of the ventral aspect of the neck, extending from the symphysis menti towards the sternum. The underlying digastric and superficial masseter muscles were separated by blunt dissection and the tendinous insertion of the deep masseteric muscle on the oblique masseteric ridge was exposed. This was then cut and reflected to expose the lateral aspect of the mandible. With a slowly running water-cooled round dental burr, defects approximately two millimetres in diameter were made in the mandible on both sides just above the masseteric ridge and in line with the distal root of the first molar. The depth of the defect was approximately one * Gamma irradiation was performed by the Nuclear Physics Research Unit of the University of the Witwatersrand
using a ${ }^{60} \mathrm{Co}$ source donated by the National Council for the Care of Cripples. 
millimetre, which ensured that it extended through the cortex into the medullary bone. This technique required only a single skin incision.

Femora-A longitudinal incision was made over the lateral aspect of the right femur. The aponeurotic insertion of the biceps femoris muscle was exposed and separated by blunt dissection. A circumscribed defect was then made in the midshaft in the femur in the same manner as described for the mandible.

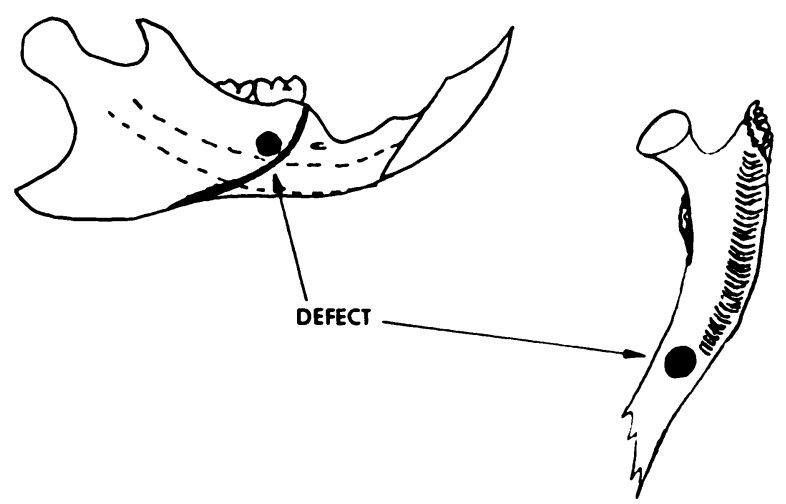

Fig. 1

Diagram showing the position of the defects in the mandible and femur.

Into all the femoral defects and into all but twelve of the mandibular defects frozen irradiated bone was transplanted. The overlying muscles were then approximated and the skin incisions sutured with silk. The recipient rats were placed in well aired cages and allowed routine laboratory rations and as much water as they desired.

Retrieval of specimens-The rats were killed in groups by coal gas, at weekly intervals up to two months and then at three-monthly intervals until nine months. The bones containing the grafts were excised en bloc together with some overlying soft tissue.

Tissue preparation-The specimens were fixed in 10 per cent buffered formalin, decalcified in either 5 per cent formic acid or de Castro's solution and embedded in paraffin wax. Serial sections were cut at 7 micra using a rotary microtome and stained with haematoxylin and eosin.

\section{RESULTS}

Mandibular defects-The healing pattern of the twelve control defects followed that described by Retief and Cleaton-Jones (1970). The defects containing the homografts showed the following healing pattern.

At one week, new bone was already in contact with the graft itself. This new bone arose from the cut margins and intact endosteal medullary bone trabeculae in the floor of the defect. No periosteal callus was seen, nor was any foreign-body reaction evident. The spaces between the new bony trabeculae were filled with a fine loose fibrous tissue (Fig. 2).

At two weeks, the endosteal callus was increased in amount, the trabeculae being larger and thicker. Periosteal callus was now evident on either side of the defect, extending over the underlying callus and graft but not completely bridging the defect. A greater area of the graft was now in contact with new bone (Fig. 3).

At three weeks, the periosteal callus had bridged the defect so that the graft was completely surrounded by bone. Remodelling of the callus had now begun and blood vessels were evident in the graft (Fig. 4). Active haemopoietic bone marrow could be seen between many of the new bony trabeculae.

VOL. 53 B, No. 3, AUgUSt 1971 
At six weeks, remodelling of the callus was continuing. Perivascular bone deposition and resorption were seen within the graft itself.

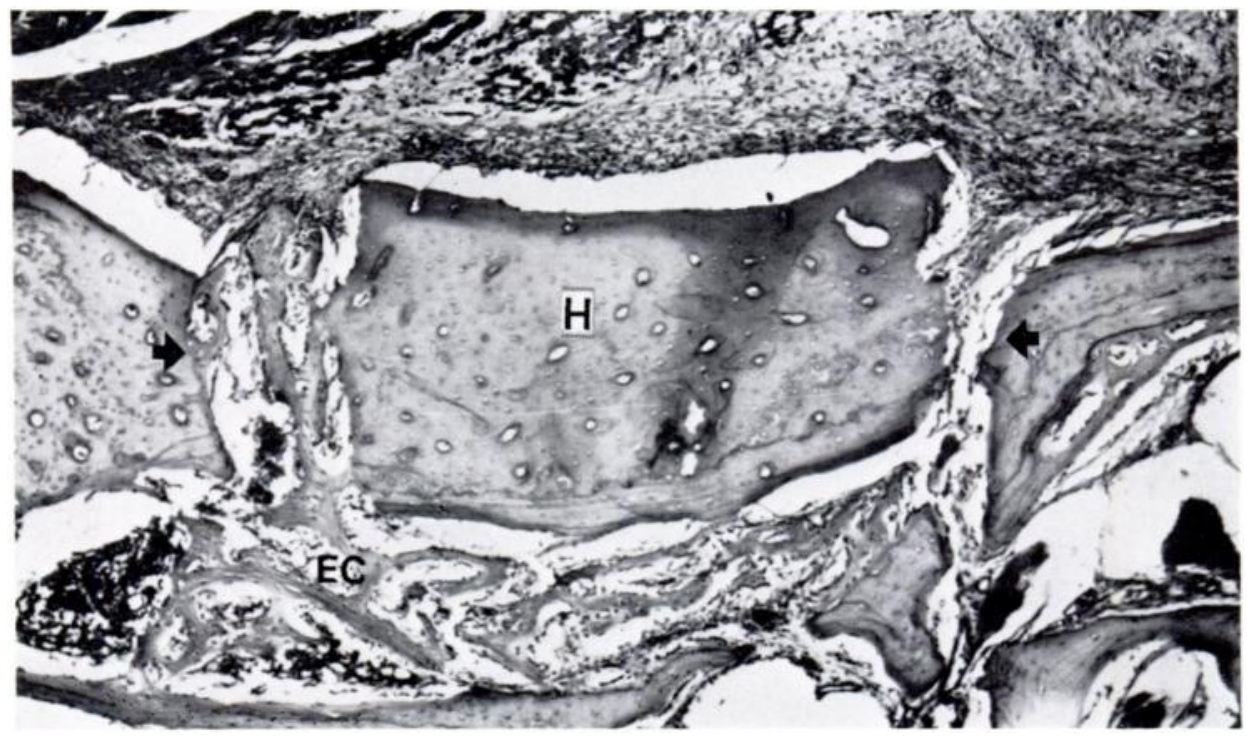

FIG. 2

Photomicrograph of homograft $(\mathrm{H})$ in a mandibular defect at one week showing endosteal callus (EC). Arrows indicate the margins of the defect. $(\therefore 40$.

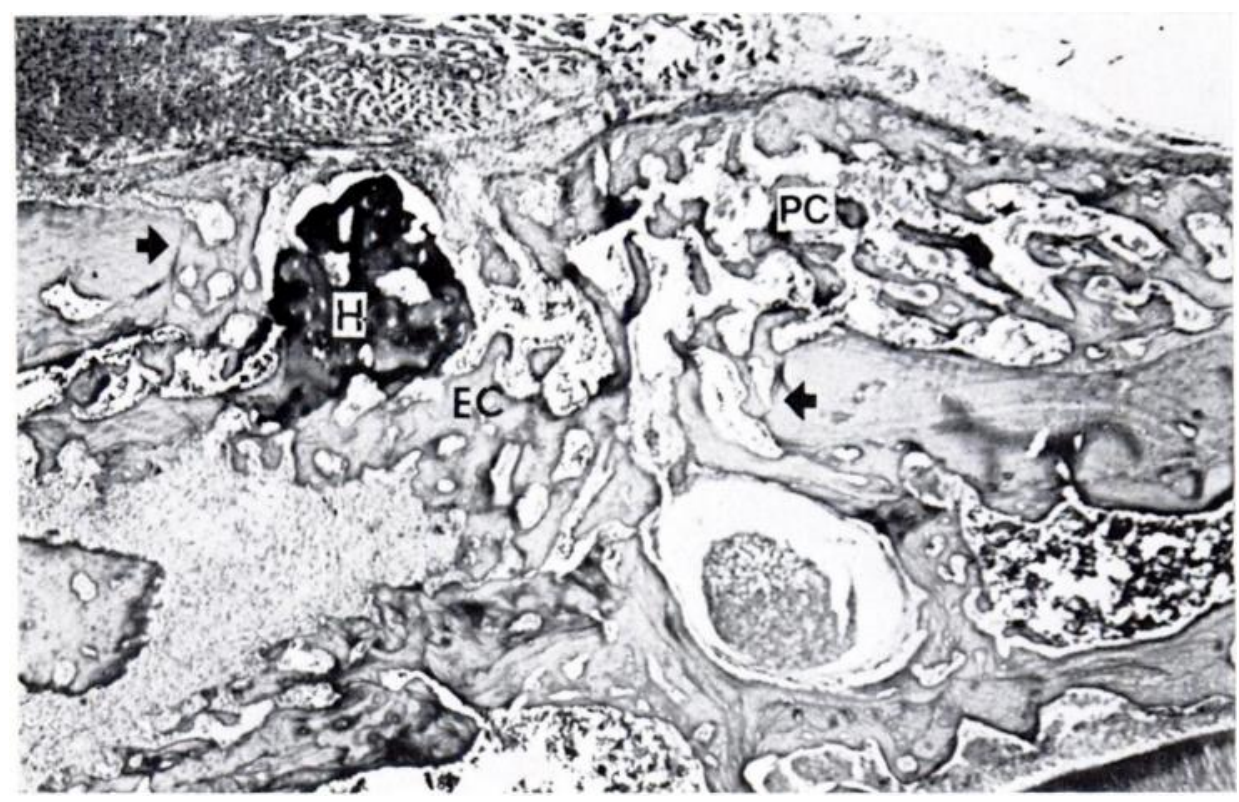

FIG. 3

Photomicrograph of homograft $(\mathrm{H})$ in a mandibular defect at two weeks showing endosteal callus $(E C)$ and periosteal callus $(P C) . \quad(\because 40$.

At two months, much remodelling had occurred, particularly of the periosteal callus, and a mature trabecular pattern was present. The grafts had now decreased in size and active bone marrow was present throughout the healing defect (Fig. 5). 
At nine months, the defect was well healed and covered with a thick cortical bone plate. The outline of the original defect could be discerned with difficulty in a number of sections. Small graft fragments were still evident in all specimens (Fig. 6).

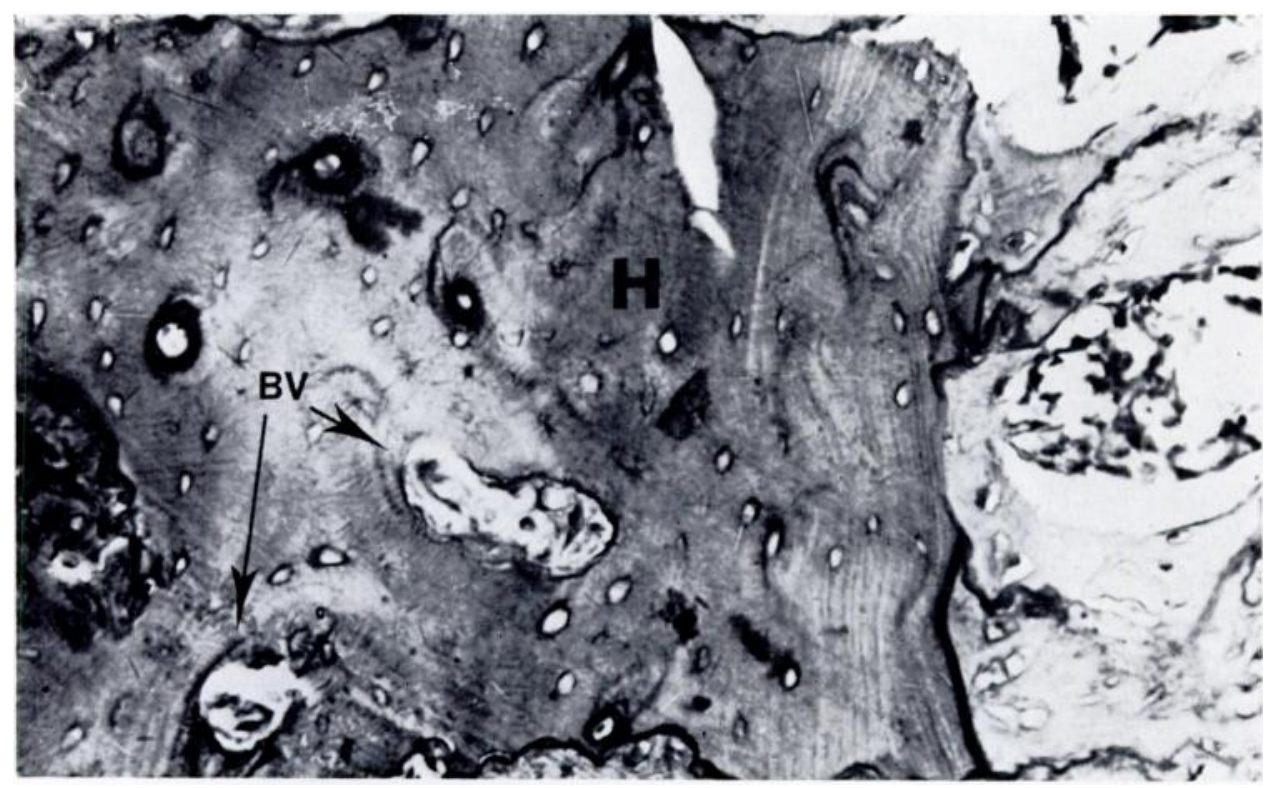

FIG. 4

High power photomicrograph of homograft $(\mathrm{H})$ at four weeks showing new blood vessels (BV). ( $\times 320$.

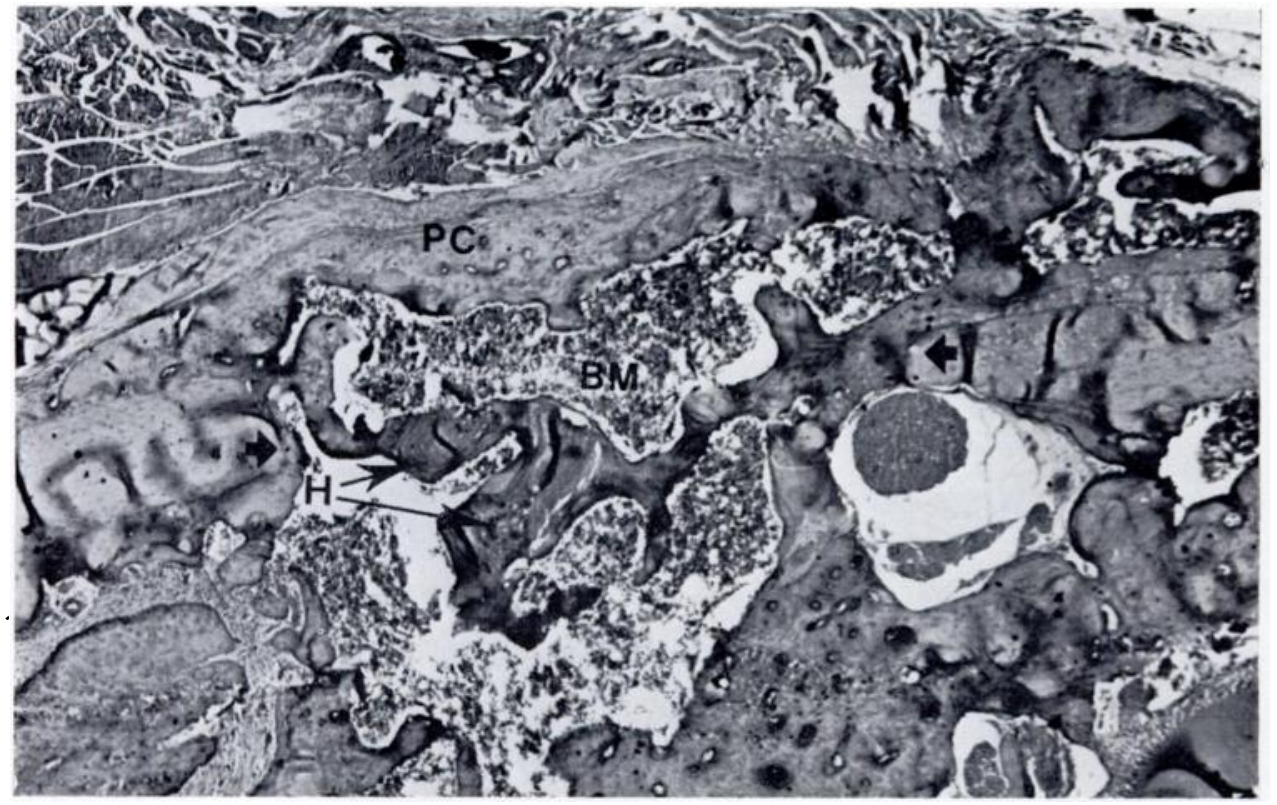

Fig. 5

Photomicrograph of homograft $(\mathrm{H})$ in mandibular defect at two months showing a thick periosteal callus (PC) and active bone marrow (BM). ( $\because 40$.

Femoral defects-The normal healing pattern of circumscribed defects in the femora of albino rats as described by Melcher and Irving (1962) was used as a control.

At one week, the graft was surrounded by a marked proliferation of callus arising from the vol. 53 B, NO. 3, AUguSt 1971 
endosteal surface and cut margins of the defect. This callus protruded outwards beyond the margin of the defect. The trabeculae were thin and orientated at right angles to the surface

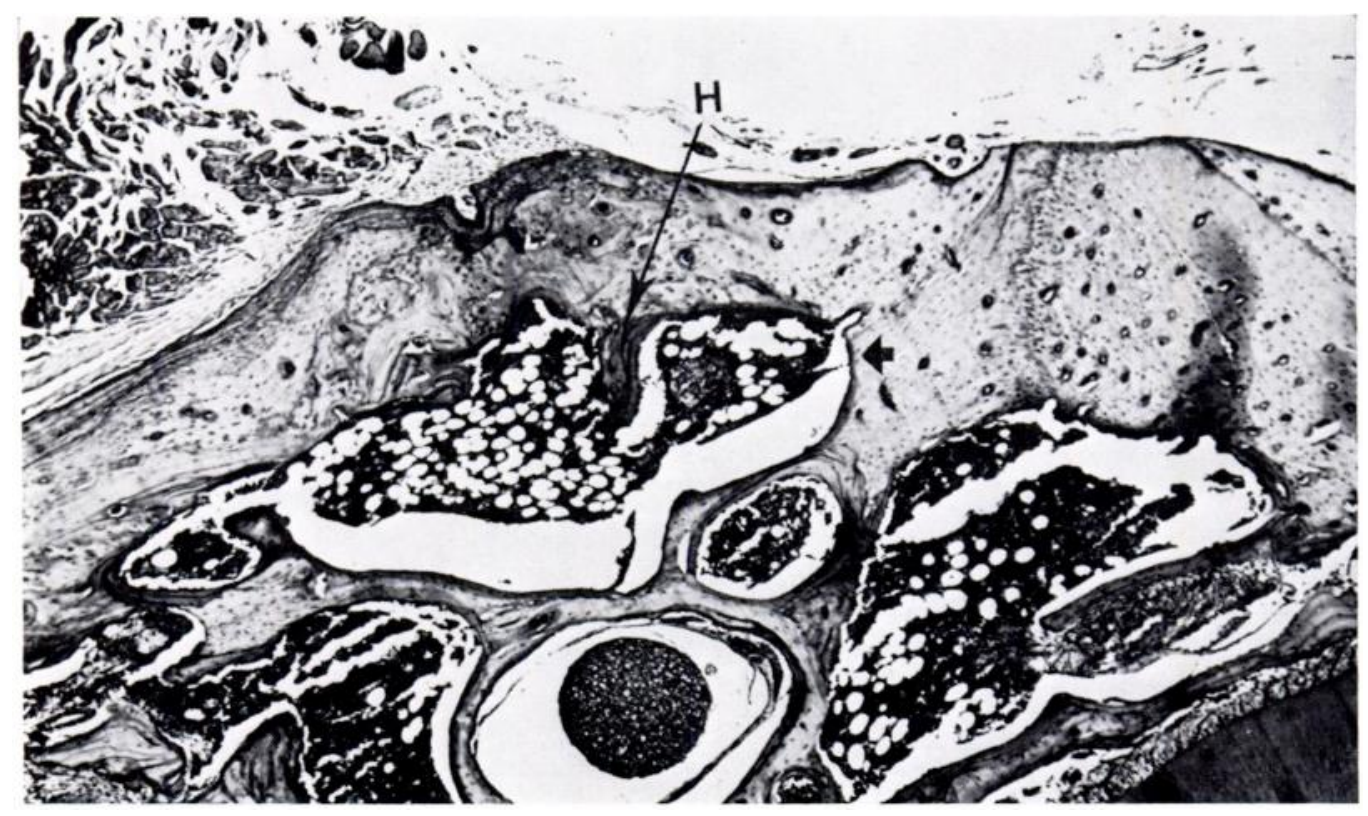

Fig. 6

Photomicrograph of a healed mandibular defect at nine months showing fragments of homograft $(\mathrm{H}) .(\times 40$.

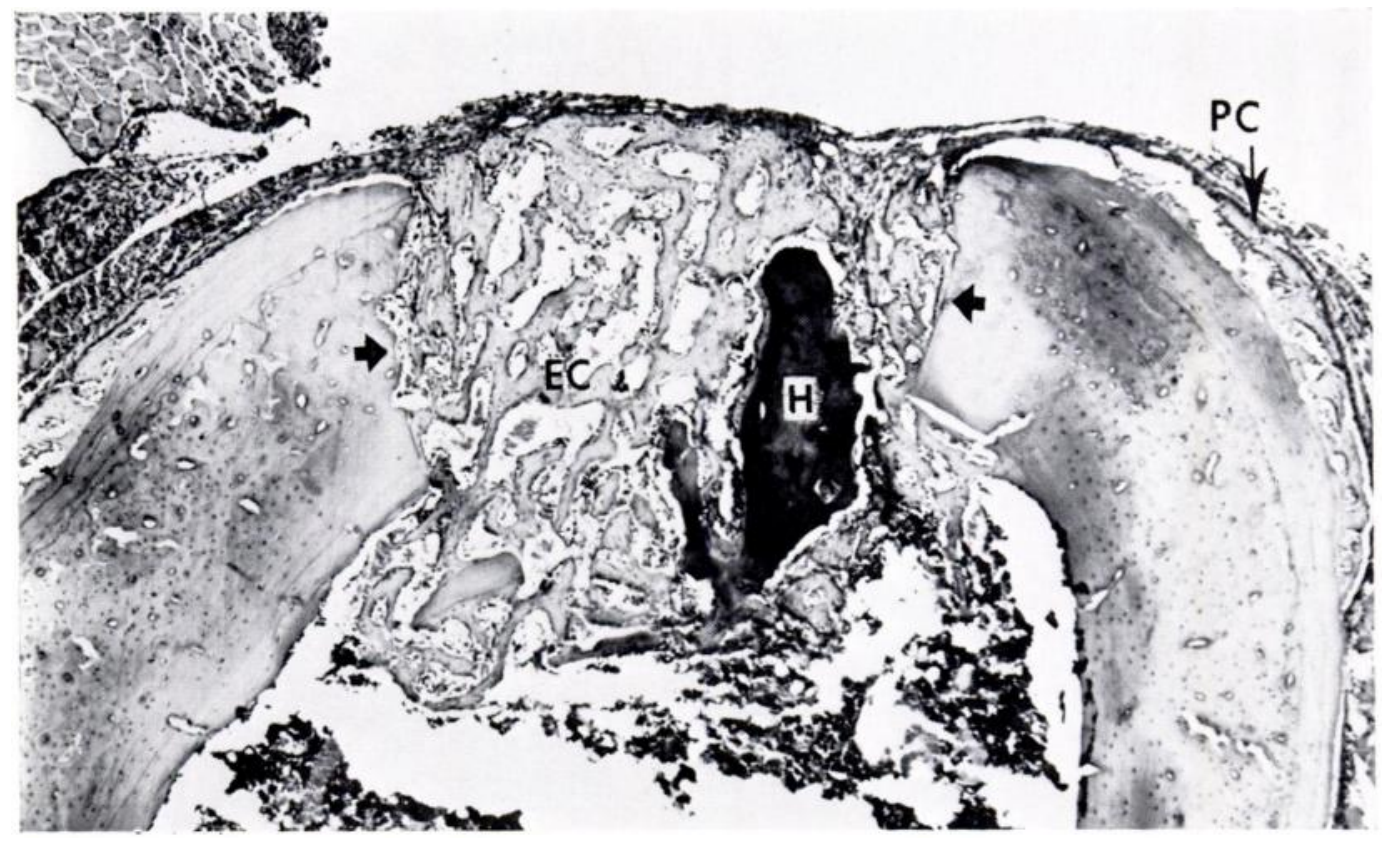

FIG. 7

Photomicrograph of homograft $(\mathrm{H})$ in a femoral defect at one week showing endosteal callus $(\mathrm{EC})$ and periosteal callus (PC). ( $\times 40$.

of the defect. A fine fibrous tissue filled the spaces between the trabeculae. New bone was laid down directly on to the surface of the graft. Subperiosteal callus was seen which was 
small in amount and well away from the edges of the defect. No foreign-body reaction was seen (Fig. 7).

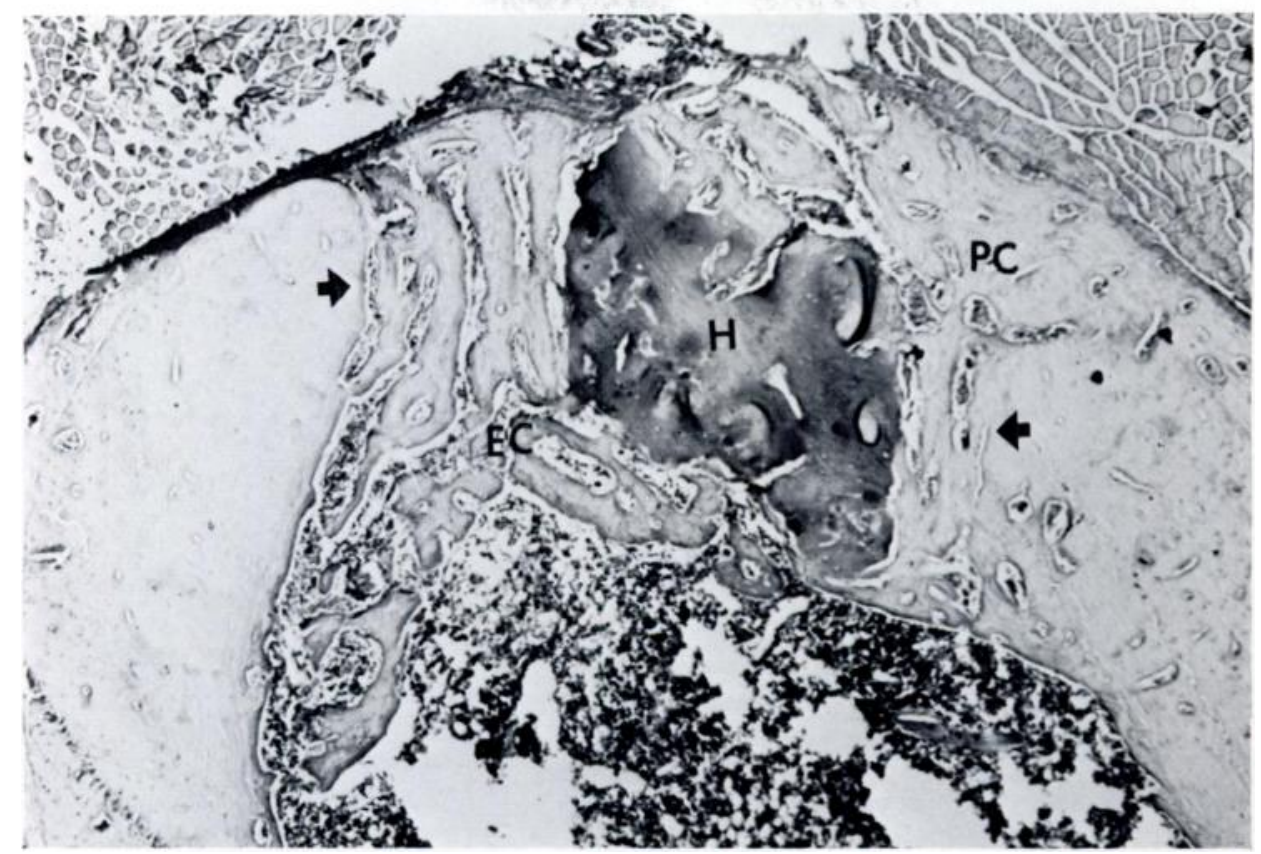

FIG. 8

Photomicrograph of homograft $(\mathrm{H})$ in a femoral defect at two weeks showing increased periosteal callus (PC) growing over thickened endosteal callus (EC). ( $\because 40$.

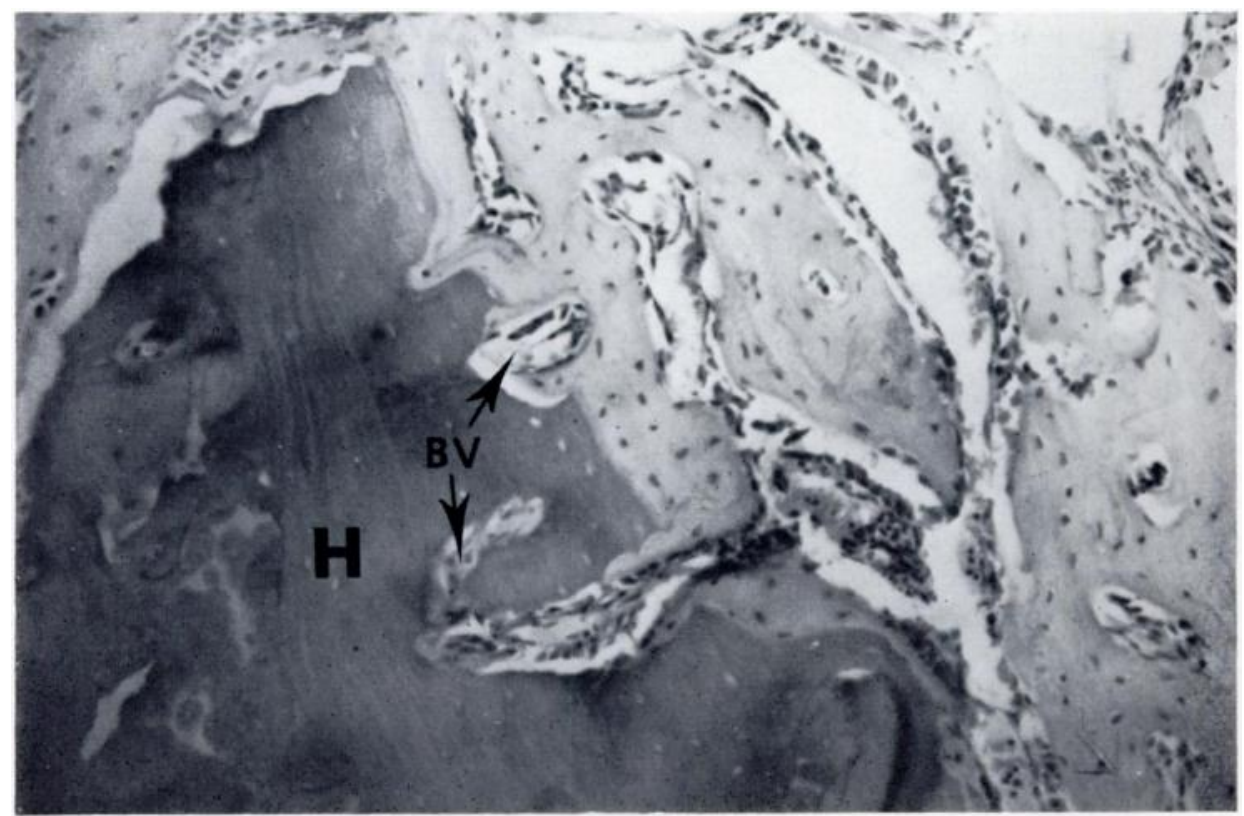

FIG, 9

High power view of the specimen shown in Figure 8. New blood vessels (BV) are seen invading the homograft $(H)$. New bone is in contact with the graft. $(\because 320$.)

At two weeks, the bony trabeculae filling the defect had increased in thickness. Periosteal callus had increased in amount and was growing over the underlying endosteal trabeculae. 
Active bone marrow was present between some of the trabeculae in the deeper layers (Fig. 8). New blood vessels were seen invading the graft. New bone was laid down directly on to all surfaces of the graft (Fig. 9).

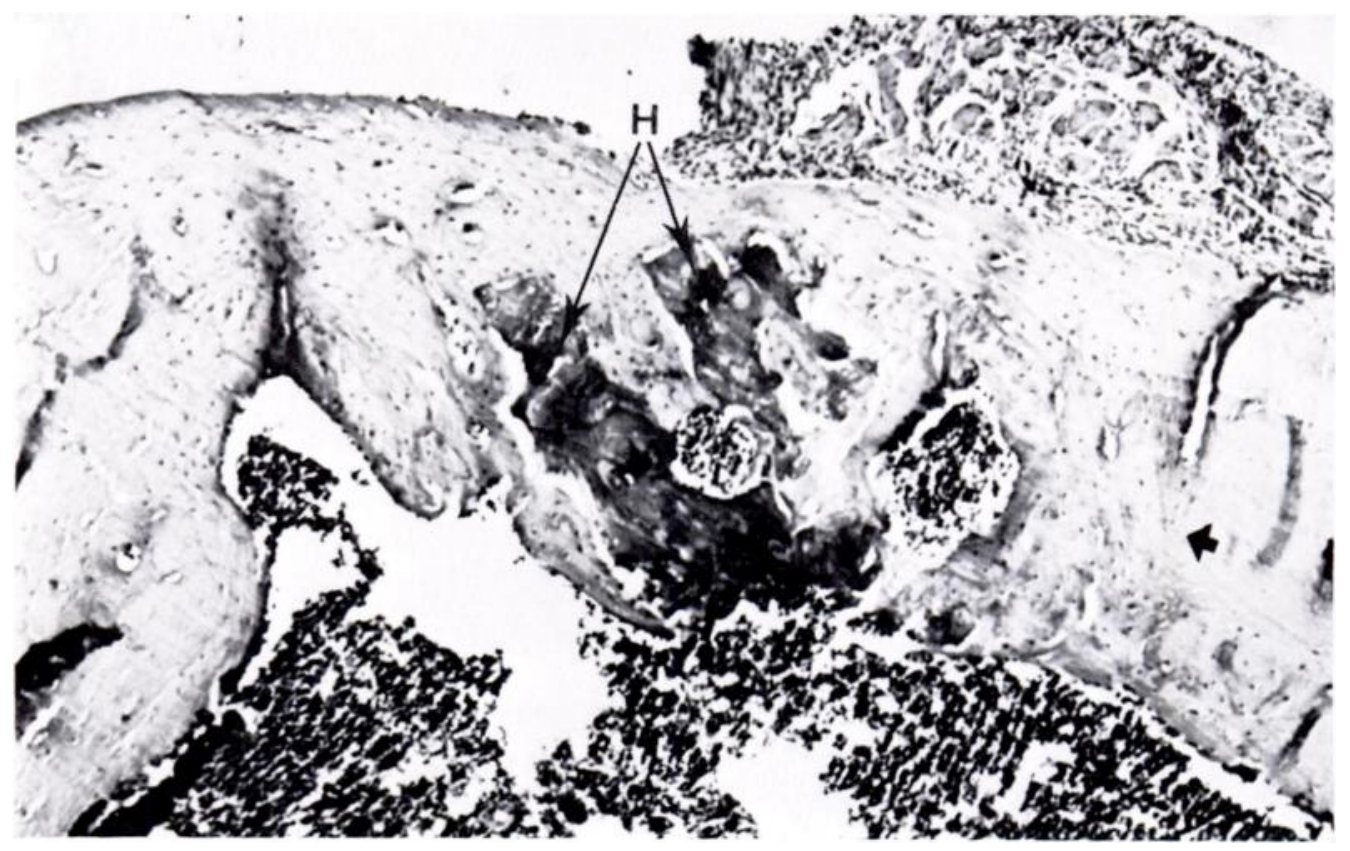

FIG. 10

Photomicrograph of homograft $(\mathrm{H})$ in a healed femoral defect at six weeks. $(\cdot 40$.

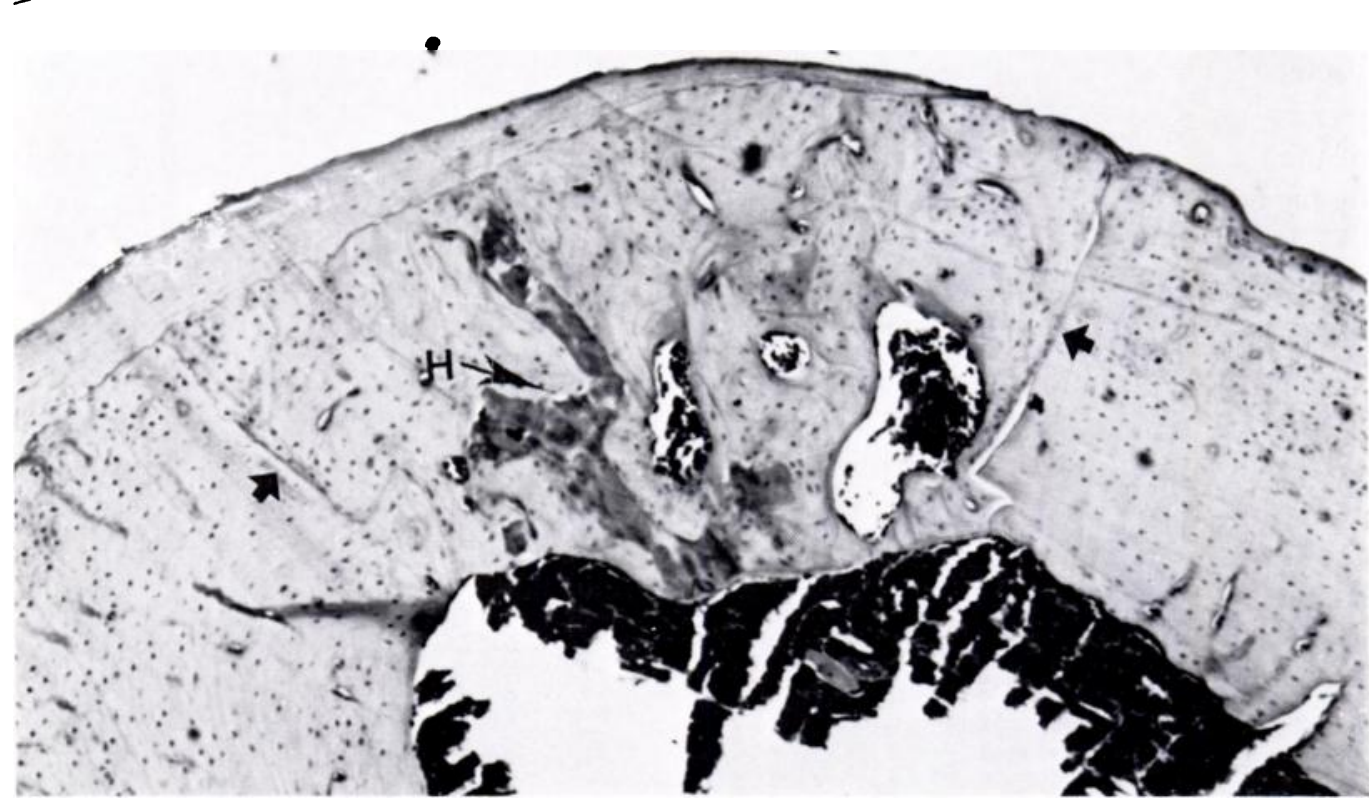

FiG. 11

Photomicrograph of a healed femoral defect at seven months showing remnants of a homograft $(\mathrm{H}) . \quad(\cdot 64$.

At six weeks, the defect was well healed with the graft incorporated into cortical bone. The graft was now much reduced in size and remodelling of the cortical plate had occurred (Fig. 10). 
At seven months, the margins of the defect were evident in some sections and small fragments of graft remained in all (Fig. 11).

\section{DISCUSSION}

An extra-oral approach to the production of the mandibular defects was used, as Mesrobian and Shklar (1969) showed that a marked inflammatory response caused by oral bacterial flora or food impaction influences healing. In the mandible careful positioning of the defect is necessary. If it is prepared too far above the masseteric ridge the underlying inferior alveolar nerve may be damaged. This adversely affects the healing pattern (Retief and Dreyer 1967). On the other hand, if the defect is made too far posteriorly above the oblique ridge then the underlying continuously erupting incisor may be damaged. Melcher (1967) showed that the defect when penetrating that tooth was not occluded by callus six weeks later. In this study any specimen showing such nerve or tooth damage was discarded.

Thompson (1958) showed that a burr speed of 500 revolutions per minute produced little degeneration of osteocytes in the bone adjoining defects prepared in the mandibles of dogs. He showed that too slow a burr speed produced fragmentation of the margins of the defect, whereas higher speeds could produce severe cellular damage. Initially this was evidenced by lighter staining of the cytoplasm and pyknosis and eventually complete disintegration of the osteocytes, leaving empty lacunae. The burr speed and water cooling used in this study produced minimal cellular damage manifested by a few empty lacunae in the bone adjoining some of the defects.

In both the mandibular and the femoral defects containing homografts no foreign-body reaction was seen and the homografts appeared well tolerated by the host tissues. These findings are similar to those of Brooks, Heiple, Herndon and Powell (1963).

The homografts did not appear in any way to inhibit osteogenesis other than serving as a mechanical barrier to the newly formed bone. The rate of new bone formation in the mandibular grafts followed closely that in the controls while the femoral grafts followed that described by Melcher and Irving (1962). It is felt therefore that the homografts in no way stimulated new bone formation. This is in keeping with the findings of Buring (1968) that gamma radiation used to sterilise organic bone matrix totally destroys the inductive capacity of the graft. The amount of new bone formed was less in amount than that in the controls by the amount of unresorbed graft.

Essentially then, our findings are similar to those of Heiple et al. (1963), who in their study of healing after the implantation of various grafts in dogs found that frozen irradiated grafts compared favourably with the controls.

\section{SUMMARY}

1. The effects of frozen irradiated homogenous bone grafts on the healing of circumscribed defects in the mandibles and femora of forty albino rats have been studied.

2. The grafts were well tolerated by the host animals and did not appear in any way either to stimulate or to inhibit osteogenesis. The rate of healing of the defects containing the homografts compared favourably with that of the control defects.

\section{REFERENCES}

Brooks, D. B., Heiple, K. G., Herndon, C. H., and Powell, A. E. (1963): Immunological Factors in Homogenous Bone Transplantation. IV. The Effect of Various Methods of Preparation and Irradiation on Antigenicity. Journal of Bone and Joint Surgery, 45-A, 1617.

BurING, K. (1968): Effects of Ionizing Radiation on the Matrix of Bone Implants. Calcified Tissue Research, 2. Supplement, 8.

BURING, K., and URIST, M. R. (1967): Effects of Ionizing Radiation on the Bone Induction Principle in the Matrix of Bone Implants. Clinical Orthopaedics and Related Research, 55, 225.

VOL. 53 B, NO. 3, AUGUST 1971 
Burwell, R. G. (1963): Studies in the Transplantation of Bone. V. The Capacity of Fresh and Treated Homografts of Bone to Evoke Transplantation Immunity. Journal of Bone and Joint Surgery, 45-B, 386.

Heiple, K. G., Chase, S. W., and Herndon, C. H. (1963): A Comparative Study of the Healing Process Following Different Types of Bone Transplantation. Journal of Bone and Joint Surgery, 45-A, 1593.

MaAtZ, R., Lentz, W., and Graf, R. (1954): Spongiosa Test of Bone Grafts for Transplantation. Journal of Bone and Joint Surgery, 36-A, 721.

Melcher, A. H. (1967): Wound Repair in the Periodontium of the Rat Incisor. Archives of Oral Biology, $12,1645$.

Melcher, A. H., and Irving, J. T. (1962): The Healing Mechanism in Artificially Created Circumscribed Defects in the Femora of Albino Rats. Journal of Bone and Joint Surgery, 44-B, 928.

Melcher, A. H., and Irving, J. T. (1963): The Effect of Implanting Various Substances into Artificially Created Circumscribed Defects in Rat Femurs. Journal of Bone and Joint Surgery, 45-B, 162.

Mesrobian, A. Z., and Shklar, G. (1969): The Effects of Dietary Zinc Sulfate Supplements on the Healing of Experimental Extraction Wounds. Oral Surgery, Oral Medicine and Oral Pathology, 28, 259.

RaINA, A. M. (1968): Experimental and Clinical Studies on X-ray Irradiated Mandibular Bone Grafts. Bulletin of Tokyo Medical and Dental University, 15, 313.

Retief, D. H., and Cleaton-Jones, P. E. (1970): The Healing of Circumscribed Defects in the Mandibles of Albino Rats. Journal of the Dental Association of South Africa, 25, 34.

Retief, D. H., and Dreyer, C. J. (1967): Effects of Neural Damage on the Repair of Bony Defects in the Rat. Archives of Oral Biology, 12, 1035.

Thompson, H. C. (1958): Effect of Drilling into Bone. Journal of Oral Surgery, 16, 22. 